\title{
Gender Differences in Pre- and Postoperative Health-Related Quality of Life Measures in Patients Who Have Had Decompression Surgery for Lumbar Spinal Stenosis
}

\author{
Yoshiomi Kobayashi ${ }^{1}$, Yoji Ogura ${ }^{1}$, Takahiro Kitagawa $^{1}$, Yoshiro Yonezawa ${ }^{1}$, \\ Yohei Takahashi ${ }^{2}$, Akimasa Yasuda ${ }^{1}$, Yoshio Shinozaki ${ }^{1}$, Jun Ogawa ${ }^{1}$ \\ ${ }^{1}$ Spine Center, Japanese Red Cross Shizuoka Hospital, Shizuoka, Japan \\ ${ }^{2}$ Department of Spine and Spinal Cord Surgery, Fujita Health University, Nagoya, Japan
}

\section{Study Design: Retrospective chart audit.}

Purpose: This study aimed to investigate the gender difference in pre- and postoperative health-related quality of life (HROOL) in patients who have had decompression surgery for lumbar spinal stenosis (LSS).

Overview of Literature: Gender differences may contribute to variations in disease presentations and health outcomes. The influence of gender on pre- and postoperative HROOL in spinal disorders remains unclear.

Methods: We reviewed 125 patients (79 men and 46 women) who had lumbar spinous process splitting laminectomy (LSPSL) for LSS. We assessed the following clinical information: Japanese Orthopedic Association (JOA) score; numerical rating scale (NRS) for low back pain (LBP), leg pain, and leg numbness; Zurich Claudication Questionnaire; JOA Back Pain Evaluation Questionnaire; RolandMorris Disability Questionnaire (RMDQ); and Short Form 8 (SF-8) as HROOL. We compared the HROOLs of men and women pre- and postoperatively.

Results: Although the preoperative NRS results for LBP were significantly higher in women $(p<0.05)$, there were no significant differences in clinical outcomes between men and women postoperatively. For HROOL, the RMDQ scores were significantly worse in women preoperatively $(p<0.05)$, but no significant differences were found postoperatively between men and women. Similarly, the SF-8 mental health score was also significantly lower in women preoperatively $(p<0.05)$, but no significant differences were noted between the two groups postoperatively.

Conclusions: LSPSL greatly reduced LBP, leg pain, and leg numbness in both genders. There were limited differences in pain and several HROOL questionnaire responses between men and women after surgery. We found that women had greater sensitivity to and/or lower tolerance for pain than men, which led to lower HRQOL mental health scores preoperatively.

Keywords: Sex difference; Patient reported outcomes; Health-related quality of life; Spinal dlsease; Laminotomy 


\section{Introduction}

Men and women differ in their health risks and surgical outcomes because of differences in physiology, psychology, and economic and social circumstances [1]. Many experimental studies have found gender differences in pain thresholds, tolerance, and responses to both pharmacological and non-pharmacological pain intervention [2-5]. Furthermore, epidemiologic and research surveys have demonstrated that pain-related symptoms occur more frequently among women than men in the general population [6,7]. However, the influence of gender on health-related quality of life (HRQOL) in spinal disorders remains unclear. Therefore, identification of the outcome differences between men and women after spinal surgery is required to better help patients in clinical practice.

Lumbar spinal stenosis (LSS) causes low back pain (LBP), leg pain, neurological deficits as manifested by functional impairment of the lower limbs and bowel and bladder dysfunctions. These symptoms could greatly affect the patients' QOL with increasing pain [8]. Even though there was a difference in the prevalence of symptomatic LSS distribution by age strata between men and women [9], nonetheless, little is known about the gender-based differences on pre- and postoperative HRQOL in LSS.

Clinical, neurological, and radiological findings have been evaluated as diagnostic parameters and outcome measures in LSS $[10,11]$. However, they could not directly reflect the outcomes, such as functional status and symptoms [8]. To overcome this problem, self-administered questionnaires (so-called patient-reported outcomes) are used to evaluate HRQOL. Although the HRQOL assessment has recently been considered to be essential in the evaluation of neurological and spinal disorders [12], the influence of gender on HRQOL after laminectomy in patients with LSS remains unclear. Hence, in this study, we analyzed data on several patient-reported outcomes for HRQOL and investigated the gender differences in preand postoperative HRQOL outcomes in patients with LSS.

\section{Materials and Methods}

\section{Patients and clinical assessments}

We retrospectively reviewed patients' medical records who had lumbar spinous process splitting laminectomy (LSPSL) [4] without fusion for LSS between June 2014 and March 2016 at Japanese Red Cross Shizuoka Hospital. LSPSL was performed in patients with no intervertebral instability (i.e., $<10^{\circ}$ of intervertebral angle change in preoperative functional radiographs and no spondylolisthesis $\geq$ grade 2), and those with scoliosis with Cobb angle $<15^{\circ}$ were selected. Patients were included if they fulfilled the following criteria: minimum 1-year follow-up and available preoperative and final follow-up questionnaires, including the Roland-Morris Disability Questionnaire (RMDQ), Japanese Orthopedic Association Back Pain Evaluation Questionnaire (JOABPEQ), Zurich Claudication Questionnaire (ZCQ), and Short Form 8 (SF-8).

The patients were divided into two groups according to gender. Data on the following were obtained: age, body mass index (BMI), estimated blood loss (EBL), time in surgery, follow-up period, and American Society of Anesthesiologists physical status classification (ASA-PS).

The Japanese Orthopedic Association (JOA) score, numerical rating scale (NRS) for LBP, leg pain, and leg numbness, and the ZCQ were evaluated preoperatively and at the final follow-up as clinical outcomes. A higher JOA score indicated better functioning. ZCQ consisted of the following: physical function, symptom severity, and patient satisfaction and was used to evaluate the severity of spinal stenosis symptoms [13]. A lower score for NRS and the ZCQ indicated a better outcome. The JOA score recovery rate was calculated as follows: (postoperative JOA score-preoperative JOA score)/(29-preoperative JOA score $) \times 100(\%)[14]$.

The JOABPEQ [15], RMDQ [16], and SF-8 Health Survey [17] were used to evaluate the HRQOL. Data on the HRQOL questionnaires administered preoperatively and at the final follow-up were obtained. The JOABPEQ scores reflected a health profile of five discrete scores calculated with its own algorithm that ranged from 0 to 100; a higher score indicated better outcome functioning. The RMDQ scores ranged from 0 to 24 , with a lower score indicating better physical functioning. The SF- 8 reflected a health profile of eight discrete scores, which were further classified as the physical component summary and mental component summary (MCS) by calculating with its own algorithm; a higher score represented better outcome functioning.

Informed consent was obtained from all participants. Our hospital institutional review board approved this study. 
Table 1. Patient demographics

\begin{tabular}{lccc} 
Characteristic & Men & Women & p-value \\
No. of cases & 79 & 46 & - \\
\hline Age $(\mathrm{yr})$ & $68.9 \pm 8.14$ & $71.9 \pm 10.9$ & 0.08 \\
\hline Body mass index $\left(\mathrm{kg} / \mathrm{m}^{2}\right)$ & $24.2 \pm 3.44$ & $22.8 \pm 3.27$ & $0.03^{*}$ \\
\hline Estimated blood loss $(\mathrm{mL})$ & $60.2 \pm 55.3$ & $51.3 \pm 54.0$ & 0.38 \\
\hline Operating time (min) & $79.0 \pm 35.0$ & $68.6 \pm 27.9$ & 0.09 \\
\hline No. of resected laminae & $1.84 \pm 0.98$ & $1.57 \pm 0.78$ & 0.11 \\
\hline Follow-up (mo) & $27.1 \pm 4.59$ & $27.4 \pm 4.43$ & 0.14 \\
\hline American Society of Anesthesiologists physical status classification & $1.94 \pm 0.43$ & $1.89 \pm 0.43$ & 0.57 \\
\hline
\end{tabular}

Values are presented as number or mean \pm standard deviation. " $p<0.05$.

\section{Surgical procedures}

The operation was performed with the patient under general anesthesia and in the prone position. Decompression was conducted by using LSPSL, as previously reported [4]. Briefly, a posterior midline skin incision was made to expose the cortex of the spinous process tip. The spinous process was drilled at the midline by using a high-speed drill with a fine 3-mm diamond-tipped bur. Thereafter, the spinous process was split to the base and detached from the lamina by using a Cobb elevator. A high-speed drill was used to remove the lamina and a Kerrison rongeur to remove the ligamentum flavum at the cranial and caudal ends of the intended laminar expansion. After the affected nerve roots and the dural sac were decompressed, the halves of the split spinous process were recapped by using a strong non-absorbable suture. The drainage tube was removed after 2 to 3 days depending on the amount of drainage. Postoperative ambulation was started without a brace on the day after the surgery.

\section{Statistical analysis}

Values are expressed as the mean \pm standard deviation. Differences in baseline characteristics were tested by using Student $t$-test for continuous variables and the chi-square test for categorical variables. Non-normally distributed variables were compared by using the Mann-Whitney non-parametric $U$-test. A $p$-value $<0.05$ was considered as indicative of statistical significance. We used GraphPad Prism software (ver. 6.0c; GraphPad Software Inc., San Diego, CA, USA) for all analyses.

\section{Results}

A total of 125 patients ( 79 men and 46 women) were eligible; the backgrounds of each group are shown in Table 1. The BMI was significantly lower in women, and there were no significant differences in age, EBL, operating time, number of resected laminae, follow-up periods, and ASA-PS between the groups (Table 1).

Although the NRS results for LBP were significantly higher in women $(p=0.02)$, no significant differences in other parameters were found preoperatively (Table 2). Moreover, there were no significant differences between men and women postoperatively in clinical outcomes, including JOA sores, JOA recovery rates, NRS, and ZCQ (Table 2). For HRQOL, no significant differences in JOABPEQ were noted between the two groups preand postoperatively (Fig. 1). LSPSL greatly reduced LBP, leg pain, and leg numbness in both genders. Although RMDQ showed a significantly higher score $(p<0.05)$ in women preoperatively, which indicates a worse outcome, no significant differences between the two groups were found postoperatively (Fig. 2). Similarly, the SF-8 MCS score was also significantly lower in women preoperatively $(p<0.05)$, and no significant differences were noted between the two groups postoperatively (Fig. 3).

\section{Discussion}

In this retrospective analysis of prospectively collected data, we showed that LSPSL greatly reduced LBP, leg pain, and leg numbness in both genders. There were limited differences in response to LSPSL in terms of pain and HRQOL between men and women. Interestingly, we also 
Table 2. Clinical outcomes according to gender

\begin{tabular}{|c|c|c|c|c|c|c|}
\hline \multirow{2}{*}{ Variable } & \multicolumn{3}{|c|}{ Preoperative } & \multicolumn{3}{|c|}{ Postoperative } \\
\hline & Men & Women & $p$-value & Men & Women & $p$-value \\
\hline JOA score & $16.5 \pm 4.56$ & $15.3 \pm 4.61$ & 0.17 & $26.1 \pm 2.72$ & $25.2 \pm 3.31$ & 0.09 \\
\hline JOA recovery rate $(\%)$ & - & - & & $76.8 \pm 20.4$ & $71.1 \pm 23.2$ & 0.16 \\
\hline \multicolumn{7}{|l|}{ Numerical rating scale } \\
\hline Low back pain & $5.35 \pm 2.70$ & $6.47 \pm 2.06$ & $0.02^{*}$ & $2.46 \pm 2.34$ & $2.71 \pm 2.24$ & 0.57 \\
\hline Leg pain & $5.70 \pm 2.84$ & $6.42 \pm 2.61$ & 0.16 & $2.00 \pm 2.30$ & $2.11 \pm 1.93$ & 0.79 \\
\hline Leg numbness & $5.53 \pm 2.77$ & $6.08 \pm 2.71$ & 0.29 & $1.95 \pm 2.15$ & $2.09 \pm 2.36$ & 0.74 \\
\hline \multicolumn{7}{|c|}{ Zurich Claudication Questionnaire } \\
\hline Physical function & $21.0 \pm 4.43$ & $22.1 \pm 5.51$ & 0.35 & $14.1 \pm 4.67$ & $14.9 \pm 4.62$ & 0.38 \\
\hline Symptom severity & $13.2 \pm 3.46$ & $13.3 \pm 3.40$ & 0.82 & $8.33 \pm 2.83$ & $8.20 \pm 2.55$ & 0.79 \\
\hline Patient satisfaction & - & - & & $11.6 \pm 4.18$ & $10.7 \pm 3.94$ & 0.22 \\
\hline
\end{tabular}

Values are presented as number or mean \pm standard deviation.

JOA, Japanese Orthopedic Association.

" $p<0.05$.

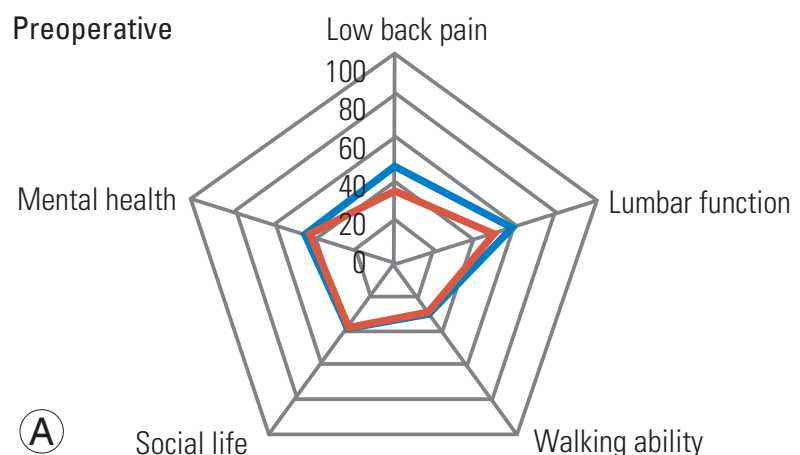

Postoperative Low back pain

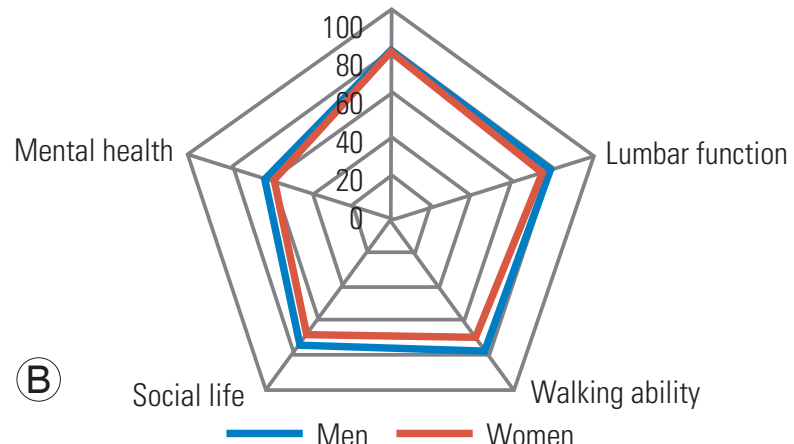

Fig. 1. (A, B) The JOABPEO discrete scores. There were no significant differences between the two groups pre- and postoperatively in the JOABPEQ. JOABPEQ, Japanese Orthopedic Association Back Pain Evaluation Questionnaire.

found that NRS for LBP, RMDQ, and SF-8 scores were significantly worse in women than in men before surgery. Previous laboratory studies have been conducted about gender differences in pain sensitivity $[3,18,19]$. Neziri et al. [3] concluded that women were more pain sensitive than

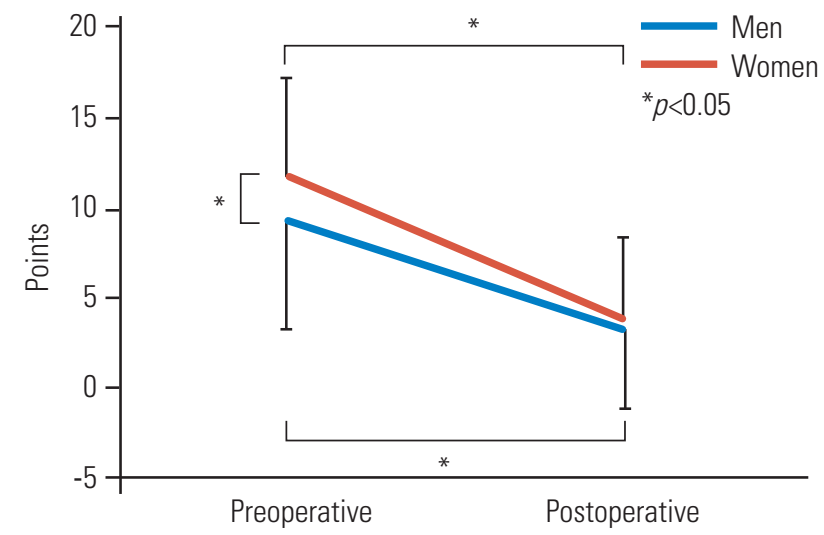

Fig. 2. Changes in RMDO between two groups after surgery. The RMD0 score was significantly higher $(p<0.05)$ in women than men preoperatively, but no significant differences between the two groups were found postoperatively. RMD0, Roland-Morris Disability Questionnaire.

were men, although the influence of gender decreased with increasing age, and another study confirmed that women were more responsive to mechanical pain stimuli [19]. This gender difference in pain perception has been explained by several mechanisms [20-22]. Symptoms, such as LBP and leg pain caused by LSS, can greatly affect a patients' QOL [8]. Therefore, in the present study, we investigated gender-based differences in pre- and postoperative HRQOL in LSS.

Regarding the clinical studies about pain, multiple reports, including a recently published review of 11,000 electronic medical records of men and women with the same diagnosis, have suggested that pain levels within 

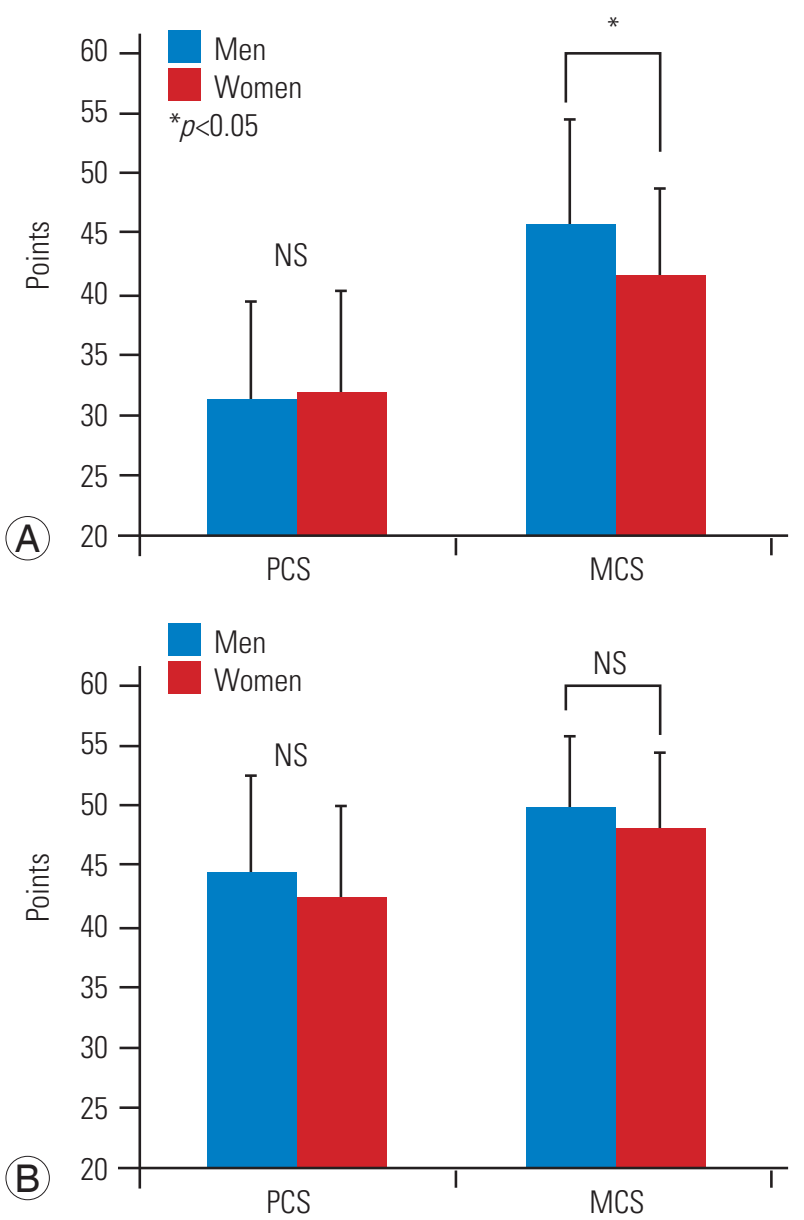

Fig. 3. The preoperative (A) and postoperative (B) PCS and MCS scores in SF-8. Although the SF-8 MCS score was also significantly lower in women than men preoperatively, no significant differences were noted between the two groups postoperatively. PCS, physical component summary; MCS, mental component summary; SF-8, Short Form 8; NS, not significant.

chronic pain are markedly higher in women than men, although the degree of stenosis may not correlate with the intensity of pain in spinal disorders, including LSS [23]. Moreover, a majority of clinical studies have suggested that women suffer pain more often (higher prevalence) for musculoskeletal, neuropathic, abdominal, and migrainerelated conditions [24]. Even in children, Stromqvist et al. [25] evaluated preoperative disability and postoperative outcome after surgery for lumbar disc herniation and found that girls reported a higher disability than did boys. Other studies have suggested that this phenomenon might reflect the tendency of women to undergo surgery at a later stage in their pathology [26]. Although it was not statistically significant, the fact that the women were on average 3 years older than the men when they presented for surgery in the present study would support this hypoth- esis if the difference is shown to be significant. Consistent with these reports, the results of this study showed the preoperative NRS results for LBP in females were significantly higher than in males. Kim et al. [27] reported that pain sensitivity was higher in women than in men according to a pain sensitivity questionnaire after adjustment for age in LSS. We found that women had more sensitivity to pain than men, which led to the higher NRS score for LBP preoperatively.

The preoperative SF- 8 mental health score also was worse in women in this study. A previous study also showed that increased pain perception for LBP and leg pain was negatively correlated with scores on HRQOL in LSS [27]. Physical pain and depression have a deeper biological connection than a simple cause and effect; the neurotransmitters that influence both pain and mood are serotonin and norepinephrine [28]. Furthermore, vague aches and LBP are often the presenting symptoms of depression, which also include chronic joint pain, limb pain, back pain, and psychomotor activity changes $[29,30]$. Nevertheless, worse physical health can worsen mental health. A previous study demonstrated that depression is more severe in patients with severe LBP than in those with mild or moderate LBP [31]. Thus, the mental health score could have been affected by chronic LBP preoperatively. Accordingly, these results suggest that women have greater sensitivity to and/or a lower tolerance for pain than that of men, which led to a lower SF-8 MCS score for women. Women might be less likely to seek medical advice until their conditions become more severe. Therefore, it is important to enlighten patients to avoid a vicious cycle of pain leading to ADL impairment that in turn leads to worsening mental health that then leads to pain.

The study strengths include the several different patient-reported outcomes that evaluate different aspects of QOL, disability, and satisfaction rate. However, this study had several limitations, including its retrospective nature. Moreover, we did not evaluate global spinal alignment, which could also affect functional outcomes and QOL. Differences in postoperative medication and rehabilitation also could have affected the clinical outcomes, which were not investigated in this study.

\section{Conclusions}

LSPSL greatly reduced LBP, leg pain, and leg numbness in both genders. None of the HRQOL questionnaires were 
significantly different between men and women postoperatively in patients with LSS, although the RMDQ and SF-8 scores were significantly worse in women preoperatively.

\section{Conflict of Interest}

No potential conflict of interest relevant to this article was reported.

\section{Author Contributions}

YK collected, and interpreted the data and wrote the initial draft of the manuscript. YO, TK, YY, YT, AY, and YS collected data. YO, YS, and JO assisted in the preparation of the manuscript. YK, YO, and JO designed the study, and critically reviewed the manuscript. All authors approved the final version of the manuscript, and agreed to be accountable for all aspects of the work in ensuring that questions related to the accuracy or integrity of any part of the work were appropriately investigated and resolved.

\section{References}

1. Greenspan JD, Craft RM, LeResche L, et al. Studying sex and gender differences in pain and analgesia: a consensus report. Pain 2007;132 Suppl 1:S26-45.

2. Bartley EJ, Fillingim RB. Sex differences in pain: a brief review of clinical and experimental findings. $\mathrm{Br}$ J Anaesth 2013;111:52-8.

3. Neziri AY, Scaramozzino P, Andersen OK, Dickenson $\mathrm{AH}$, Arendt-Nielsen L, Curatolo M. Reference values of mechanical and thermal pain tests in a pain-free population. Eur J Pain 2011;15:376-83.

4. Watanabe K, Matsumoto M, Ikegami T, et al. Reduced postoperative wound pain after lumbar spinous process-splitting laminectomy for lumbar canal stenosis: a randomized controlled study. J Neurosurg Spine 2011;14:51-8.

5. Musey PI Jr, Linnstaedt SD, Platts-Mills TF, et al. Gender differences in acute and chronic pain in the emergency department: results of the 2014 Academic Emergency Medicine consensus conference pain section. Acad Emerg Med 2014;21:1421-30.

6. Fillingim RB, Doleys DM, Edwards RR, Lowery D. Clinical characteristics of chronic back pain as a function of gender and oral opioid use. Spine (Phila Pa 1976) 2003;28:143-50.
7. Andersson HI, Ejlertsson G, Leden I, Rosenberg C. Chronic pain in a geographically defined general population: studies of differences in age, gender, social class, and pain localization. Clin J Pain 1993;9:174-82.

8. Padua L, Padua R, Mastantuoni G, Pitta L, Caliandro P, Aulisa L. Health-related quality of life after surgical treatment for lumbar stenosis. Spine (Phila Pa 1976) 2004;29:1670-4.

9. Ishimoto Y, Yoshimura N, Muraki S, et al. Prevalence of symptomatic lumbar spinal stenosis and its association with physical performance in a populationbased cohort in Japan: the Wakayama Spine Study. Osteoarthritis Cartilage 2012;20:1103-8.

10. Lurie JD, Birkmeyer NJ, Weinstein JN. Rates of advanced spinal imaging and spine surgery. Spine (Phila Pa 1976) 2003;28:616-20.

11. Leinonen V, Maatta S, Taimela S, et al. Paraspinal muscle denervation, paradoxically good lumbar endurance, and an abnormal flexion-extension cycle in lumbar spinal stenosis. Spine (Phila Pa 1976) 2003;28:324-31.

12. Zanoli G, Romanini E, Padua R, Traina GC, Massari L. EBM in musculoskeletal diseases: where are we? Acta Orthop Scand Suppl 2002;73:4-7.

13. Stucki G, Daltroy L, Liang MH, Lipson SJ, Fossel AH, Katz JN. Measurement properties of a self-administered outcome measure in lumbar spinal stenosis. Spine (Phila Pa 1976) 1996;21:796-803.

14. Chiba K, Toyama Y, Matsumoto M, Maruiwa H, Watanabe M, Nishizawa T. Intraspinal cyst communicating with the intervertebral disc in the lumbar spine: discal cyst. Spine (Phila Pa 1976) 2001;26:2112-8.

15. Fukui M, Chiba K, Kawakami M, et al. JOA Back Pain Evaluation Questionnaire (JOABPEQ)/JOA Cervical Myelopathy Evaluation Questionnaire (JOACMEQ): the report on the development of revised versions. April 16, 2007. The Subcommittee of the Clinical Outcome Committee of the Japanese Orthopaedic Association on Low Back Pain and Cervical Myelopathy Evaluation. J Orthop Sci 2009;14:34865.

16. Roland M, Morris R. A study of the natural history of low-back pain: part II: development of guidelines for trials of treatment in primary care. Spine (Phila Pa 1976) $1983 ; 8: 145-50$. 
17. Turner-Bowker DM, Bayliss MS, Ware JE Jr, Kosinski M. Usefulness of the SF-8 Health Survey for comparing the impact of migraine and other conditions. Qual Life Res 2003;12:1003-12.

18. Sibille KT, Kindler LL, Glover TL, et al. Individual differences in morphine and butorphanol analgesia: a laboratory pain study. Pain Med 2011;12:1076-85.

19. Kroner-Herwig B, Gassmann J, Tromsdorf M, Zahrend E. The effects of sex and gender role on responses to pressure pain. Psychosoc Med 2012;9:Doc01.

20. Racine M, Tousignant-Laflamme Y, Kloda LA, Dion D, Dupuis G, Choiniere M. A systematic literature review of 10 years of research on sex/gender and experimental pain perception: part 1: are there really differences between women and men? Pain 2012;153:602-18.

21. Racine M, Tousignant-Laflamme Y, Kloda LA, Dion D, Dupuis G, Choiniere M. A systematic literature review of 10 years of research on sex/gender and pain perception: part 2: do biopsychosocial factors alter pain sensitivity differently in women and men? Pain 2012;153:619-35.

22. Unruh AM. Gender variations in clinical pain experience. Pain 1996;65:123-67.

23. Ruau D, Liu LY, Clark JD, Angst MS, Butte AJ. Sex differences in reported pain across 11,000 patients captured in electronic medical records. J Pain 2012;13:228-34.
24. Fillingim RB, King CD, Ribeiro-Dasilva MC, RahimWilliams B, Riley JL 3rd. Sex, gender, and pain: a review of recent clinical and experimental findings. J Pain 2009;10:447-85.

25. Stromqvist F, Stromqvist B, Jonsson B, Gerdhem P, Karlsson MK. Lumbar disc herniation surgery in children: outcome and gender differences. Eur Spine J 2016;25:657-63.

26. Katz JN, Wright EA, Guadagnoli E, Liang MH, Karlson EW, Cleary PD. Differences between men and women undergoing major orthopedic surgery for degenerative arthritis. Arthritis Rheum 1994;37:68794.

27. Kim HJ, Suh BG, Lee DB, et al. Gender difference of symptom severity in lumbar spinal stenosis: role of pain sensitivity. Pain Physician 2013;16:E715-23.

28. Trivedi MH. The link between depression and physical symptoms. Prim Care Companion J Clin Psychiatry 2004;6(Suppl 1):12-6.

29. Lyness JM, Caine ED, King DA, Cox C, Yoediono Z. Psychiatric disorders in older primary care patients. J Gen Intern Med 1999;14:249-54.

30. Freburger JK, Holmes GM, Agans RP, et al. The rising prevalence of chronic low back pain. Arch Intern Med 2009;169:251-8.

31. Marcic M, Mihalj M, Ivica N, Pintaric I, Titlic M. How severe is depression in low back pain patients? Acta Clin Croat 2014;53:267-71. 